\title{
XMM-Newton observation of the Seyfert 1 ESO 198-G24
}

\author{
D. Porquet ${ }^{1}$, J. S. Kaastra ${ }^{2}$, K. L. Page ${ }^{3}$, P. T. O’Brien ${ }^{3}$, M. J. Ward ${ }^{3}$, and J. Dubau ${ }^{4}$ \\ 1 Max-Planck-Institut für extraterrestrische Physik, Postfach 1312, 85741 Garching, Germany \\ 2 SRON, Sorbonnelaan 23584 CA Utrecht, The Netherlands \\ 3 X-Ray Astronomy Group; Department of Physics and Astronomy, Leicester University, LE1 7RH, UK \\ ${ }^{4}$ LIXAM, Université Paris-Sud, 91405 Orsay Cedex, France
}

Received 20 February 2003 / Accepted 7 October 2003

\begin{abstract}
We present the results from an XMM-Newton observation (January 24, 2001) of the bright Seyfert 1 galaxy ESO 198-G24 $(z=0.045)$. We found that this Seyfert has an intrinsic $2-10 \mathrm{keV}$ luminosity of about $10^{44} \mathrm{erg} \mathrm{s}^{-1}$. This source shows no intrinsic absorption in addition to the Galactic absorption $\left(\mathcal{N}_{\mathrm{H}} \sim 3 \times 10^{20} \mathrm{~cm}^{-2}\right)$. We found both with EPIC and RGS that this source possesses significantly steeper spectra below $\sim 1.5-2 \mathrm{keV}$ than observed at higher X-ray energies, the so-called soft X-ray excess. The RGS spectra reveal no significant narrow absorption lines suggesting that if there is a warm absorber, it either has a relatively low column density, or a very high ionization parameter. The RGS data are well described by the com-

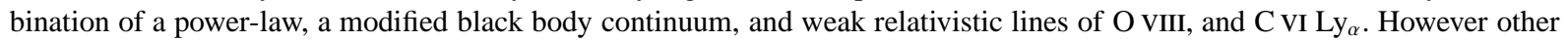
interpretations are not definitely excluded. The $2-10 \mathrm{keV}$ energy band is well fitted by an absorbed power-law with a photon spectral index of $\Gamma=1.77 \pm 0.04$ (consistent with the typical $\Gamma \sim 1.7$ found in Seyfert 1 galaxies). We found the presence of a narrow Gaussian emission line at $6.41 \mathrm{keV}$ (i.e. $<\mathrm{Fe}$ XVII) with a moderate equivalent width of about $60-70 \mathrm{eV}$, and we found an upper limit for a broad component, if any, of $75 \mathrm{eV}$. We also found a weak absorption edge associated with cold iron with an optical depth of about 0.2 .
\end{abstract}

Key words. galaxies: active - quasars: individual: ESO 198-G24 - X-rays: galaxies

\section{Introduction}

The Seyfert 1 ESO 198-G24 $(z=0.045)$ is one object of the Piccinotti Sample of Active Galactic Nuclei (Piccinotti et al. 1982). It was already observed in the soft X-ray band by the ROSAT PSPC (Turner et al. 1993) during two distinct observations (December 1991 and July 1992). This source was selected because of a low absorbing column density $\left(\mathcal{N}_{\mathrm{H}} \sim\right.$ $3 \times 10^{20} \mathrm{~cm}^{-2}$ ) along the line-of-sight. It was found that this source possesses a significantly steeper spectrum below $\sim 1 \mathrm{keV}$ than observed at higher X-ray energies. During the ROSAT December observation, the soft X-ray spectrum of ESO 198-G24 suggested a spectral emission or absorption feature, either a Gaussian emission line at energy $0.75 \mathrm{keV} \pm$ $0.04 \mathrm{keV}$ with an equivalent width (EW) of $99 \pm 30 \mathrm{eV}$ (most readily identified as a blend of emission species dominated by ionized iron and oxygen), or an absorption edge at $E=$ $1.16 \pm 0.06 \mathrm{keV}(\tau=0.37 \pm 0.16)$. On the contrary during the ROSAT July observation, the spectrum with a similar signalto-noise ratio was adequately described by a simple featureless absorbed power-law model. Turner et al. (1993) noted that the spectral feature appeared in the brighter state observation of

Send offprint requests to: D. Porquet,

e-mail: dporquet@mpe.mpg.de
ESO 198-G24, as might be expected if it originates in material responding to ionization by the active nucleus. The only constraint on the variability time scale was that it must be less than 6 months. ESO 198-G24 was observed at higher energies by BATSE on board CGRO (Malizia et al. 1999), and was reported for the first time as a hard X-ray emitting source: $F_{\text {MEAN }}(2-100 \mathrm{keV})=5.27 \times 10^{-11} \mathrm{erg} \mathrm{cm}^{-2} \mathrm{~s}^{-1}$ (weighted mean calculated over nearly 4 years of observations). In the $2-10 \mathrm{keV}$ energy band a variability factor of 1.31 was observed, with a mean unabsorbed flux of $3.3 \times 10^{-11} \mathrm{erg} \mathrm{cm}^{-2} \mathrm{~s}^{-1}$.

Very recently, Guainazzi (2003) presented results from ASCA (July 8th, 1997; 40 ks), XMM-Newton (December 1st, 2000; $~ 9 \mathrm{ks}$, guaranteed time), and BeppoSAX (January 23th, 2001 ; $\sim 150 \mathrm{ks}$ ) data. He focused on the study of the $\mathrm{Fe} \mathrm{K}_{\alpha}$ line, and found that the intensity and line profile are different between these three observations. During the ASCA observation the line profile at $6.4 \mathrm{keV}$ is narrow without any indication of a broad component. On the contrary the XMM-Newton $\mathrm{PN}$ spectrum (effective time duration of about $6.8 \mathrm{ks}$ ) displayed a broader line centered at $6.4 \mathrm{keV}$ with an additional weak feature at $5.7 \mathrm{keV}$. Guainazzi suggested that this may be one example of "double-horned profile", similarly to the Seyfert 1 MCG-6-30-15 (e.g., Tanaka et al. 1995; Wilms et al. 2001). 
We present here the observation of ESO 198-G24 in January 24th, 2001 obtained with the X-ray satellite XMMNewton. This present work is the first analysis for this object combining moderate (EPIC) and high resolution data (RGS). Section 2 details the observation and data reduction procedures. Section 3 reports the spectral analysis of the EPIC data ( $2-5 \mathrm{keV}$ continuum, the $\mathrm{Fe} \mathrm{K}_{\alpha}$ line region, and the broad band $0.3-10 \mathrm{keV}$ energy band) and of the RGS data. The results are discussed in Sect. 4.

\section{XMM-Newton observation and data analysis}

ESO 198-G24 was observed by XMM-Newton on January 24, 2001 (AO-1, $30 \mathrm{ks}$ duration). The observations with the EPIC MOS (Turner et al. 2001), PN (Strüder et al. 2001) and the RGS (den Herder et al. 2001) detectors were done. There are no other strong X-ray sources seen within the field of view. The MOS and PN cameras (EPIC) operated in standard Full Frame mode using the medium filter. The data are screened and re-processed with the XMM SAS (Science Analysis Software), version 5.3.3. The EPIC data cleaning was improved by rejecting solar flares. After this data cleaning, we obtain as net exposure times about $22.9 \mathrm{ks}, 23.1 \mathrm{ks}$, and $15.6 \mathrm{ks}$ for MOS1, MOS2, and PN respectively. Only X-ray events corresponding to single events (i.e. pattern 0) are used for both MOS and $\mathrm{PN}$, in order to reach the best spectral resolution, and limit effect of pile-up. We note for further comparison that Guainazzi (2003) used single and double events for spectral analysis of the December 2000 XMM-Newton observation. There was negligible flux variability of ESO 198-G24 $(<10 \%)$ during the present observation, therefore we use the cumulative data for all our subsequent analyses. We then proceed to extract spectra for both source and background for each EPIC detector. A circular source region is defined around the centroid position of ESO 198-G24, of 1' radius for the MOS, and of $40^{\prime \prime}$ for the PN (to avoid the edge of the chip). The majority of source counts fall onto the source regions (at least 95\%). Background spectra are taken from an annular radius centered on ESO 198-G24, between 3.2' and 5.2' (excluding $\mathrm{X}$-ray point sources). The background subtracted spectra are fitted, using XSPEC v11.2.0. We use the following response matrices: m1_medv9q19t5r5_p0_15.rsp, m2_medv9q19t5r5_p0_15.rsp, epn_ff20_sY9_medium.rsp. The spectra are binned to a minimum of 20 counts per bin to apply the $\chi^{2}$ minimalisation technique.

Both RGS cameras are operated in the standard spectroscopy + Q mode. The RGS data are improved by rejecting solar flares. After this data cleaning, we obtain as net exposure time about $24.2 \mathrm{ks}$ and $23.6 \mathrm{ks}$ for respectively RGS1 and RGS2.

Further on, values of $H_{0}=50 \mathrm{~km} \mathrm{~s}^{-1} \mathrm{Mpc}^{-1}$ and $q_{0}=0$ are assumed. All the fit parameters are given in the galaxy restframe. The errors quoted correspond to $90 \%$ confidence ranges for one interesting parameter $\left(\Delta \chi^{2}=2.71\right)$. The cross-sections for X-ray absorption by the interstellar medium used throughout this work are from Wilms et al. (2000), and the element abundances are from Anders \& Grevesse (1989).

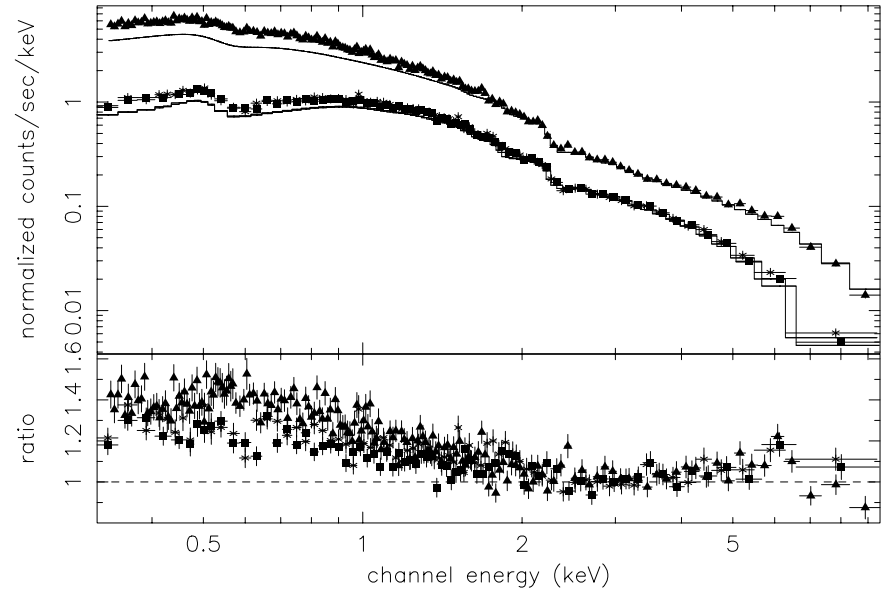

Fig. 1. The EPIC spectra of ESO 198-G24 (observer frame and binning at $20 \sigma)$. An absorbed power-law $(\Gamma \sim 1.77)$ has been fitted within the $2-5 \mathrm{keV}$ energy range, and extrapolated to lower and higher energies. A broad soft X-ray excess is clearly seen extending to almost $1.5-2 \mathrm{keV}$, as well a deviation near $6.2 \mathrm{keV}$. Filled squares: MOS1, stars: MOS2, and triangles: PN.

\section{Spectral analysis}

\subsection{The EPIC data}

At a first step, we fit the EPIC data with an absorbed powerlaw over the $0.3-10 \mathrm{keV}$ bandpass. We do not obtain an acceptable fit with a single absorbed power-law: $\Gamma=1.92 \pm 0.01$ $\left(\chi^{2} /\right.$ d.o.f. $=1825.341 / 1537$, with d.o.f. degrees of freedom $)$. We do not find any additional intrinsic absorbing medium to the Galactic column density, i.e. $3.09 \times 10^{20} \mathrm{~cm}^{-2}$ (obtained with the tool COLDENS ${ }^{1}$ ). Therefore further on, we fix the column density to the above Galactic value. We split our following spectral analysis in several energy bands: $2-5 \mathrm{keV}$, the $\mathrm{Fe} \mathrm{K}_{\alpha}$ line region, the $0.3-10 \mathrm{keV}$ broad band energy including the soft excess feature.

\subsubsection{The $2-5 \mathrm{keV}$ energy band}

We begin studying the spectra by fitting an absorbed powerlaw model to the EPIC spectra in the $2-5 \mathrm{keV}$ energy band, where the spectrum should be relatively unaffected by possible presence of a soft excess or a Warm Absorber-Emitter medium, of $\mathrm{Fe}_{\alpha}$ line emission, and of a contribution above $8 \mathrm{keV}$ that could be associated with a high energy reflection hump. The data are very well fitted by a single power-law model with $\Gamma=1.77 \pm 0.04\left(\chi^{2} /\right.$ d.o.f. $\left.=681.3 / 713\right)$. This photon index value is entirely consistent with those found by Guainazzi (2003) for different time observations (in order of increasing 2-10 keV flux): $1.75_{-0.03}^{+0.05}$ (ASCA, July 1997), $1.77 \pm 0.03$ (XMM-Newton, December 2000), and $1.79 \pm 0.04$ (BeppoSAX, January 2001). Figure 1 displays the data to model ratio extrapolated over the $0.3-10 \mathrm{keV}$ broad band energy. A significant positive residual is clearly seen below $1.5-2 \mathrm{keV}$, both in MOS and PN data. This could be due to the presence of a soft excess due for example to either

\footnotetext{
1 http://asc.harvard.edu/toolkit/colden.jsp
} 
Table 1. Fit of the EPIC data in the $2-10 \mathrm{keV}$ energy range. $E$ is the energy (in keV) of the corresponding emission line or absorption edge. $E W$ is the equivalent with of the emission line in eV. $\tau$ is the optical depth of the absorption edge. $F$-test is the $F$-test probability adding one component compared to the single power-law model.

\begin{tabular}{cccccccc}
\hline \hline Model & $\Gamma$ & $\begin{array}{c}E_{\text {line }} \text { or } E_{\text {edge }} \\
(\text { in } \mathrm{keV})\end{array}$ & $\begin{array}{c}\sigma_{\text {line }} \\
(\mathrm{eV})\end{array}$ & $\begin{array}{c}F_{\text {line }} \\
\left(\mathrm{erg} \mathrm{cm}^{-2} \mathrm{~s}^{-1}\right)\end{array}$ & $\begin{array}{c}E W_{\text {line }}(\mathrm{eV}) \\
\tau_{\text {edge }}\end{array}$ & $F$-test & $\chi^{2} /$ d.o.f. \\
\hline PL & $\Gamma=1.77 \pm 0.03$ & - & - & - & - & - & $986.0 / 973$ \\
\hline PL+ GA & $\Gamma=1.78 \pm 0.03$ & $6.41_{-0.04}^{+0.02}$ & 10 (fixed) & $9.5 \pm 2.9 \times 10^{-6}$ & $74 \pm 23$ & $99.99 \%$ & $956.1 / 971$ \\
\hline PL+GA & $\Gamma=1.78 \pm 0.03$ & $6.40 \pm 0.04$ & $57_{-57}^{+55}$ & $1.11_{-0.39}^{+0.43} \times 10^{-5}$ & $86_{-30}^{+33}$ & $99.99 \%$ & $955.1 / 970$ \\
\hline PL+ GA & $\Gamma=1.77 \pm 0.03$ & 5.7 (fixed) & 10 (fixed) & $<1.2 \times 10^{-5}$ & $<23$ & $40 \%$ & $985.7 / 972$ \\
\hline PL + GA & $\Gamma=1.77 \pm 0.03$ & $6.92 \pm 0.08$ & 10 (fixed) & $4.2 \pm 2.8 \times 10^{-6}$ & $37 \pm 25$ & $94.8 \%$ & $980.0 / 971$ \\
\hline PL+ edge & $\Gamma=1.72 \pm 0.03$ & $7.25_{-0.13}^{+0.21}$ & - & - & $0.21 \pm 0.08$ & $99.97 \%$ & $964.3 / 971$ \\
\hline PL + 2×GA & $\Gamma=1.75 \pm 0.03$ & $6.41 \pm 0.03$ & 10 (fixed) & $8.8 \pm 2.9 \times 10^{-6}$ & $68 \pm 22$ & - & $935.7 / 967$ \\
& & $6.92 \pm 0.10$ & 10 (fixed) & $3.6 \pm 2.7 \times 10^{-6}$ & $37 \pm 25$ & & \\
+ edge & & $7.24_{-0.14}^{+0.25}$ & - & - & $0.17 \pm 0.07$ & & \\
\hline
\end{tabular}

emission from a cold/ionized accretion disk, or complex absorption and/or emission from highly ionized gas, the so-called "Warm Absorber" (WA). There is also a positive deviation near $6.2 \mathrm{keV}$ (in the observer frame), suggesting the presence of an $\mathrm{Fe} \mathrm{K}_{\alpha}$ complex emission line.

\subsubsection{The $\mathrm{FeK}_{\alpha}$ line}

The $\mathrm{Fe}_{\alpha}$ emission line near $6.4 \mathrm{keV}$ was found to be a common feature in the hard X-ray spectra of many broad-line Seyfert 1 galaxies (e.g., Pounds et al. 1990; Nandra \& Pounds 1994). It has been recognized as a powerful diagnostic of the innermost regions of AGN.

In the $2-10 \mathrm{keV}$ energy band, a simple absorbed powerlaw model gives a good fit (see Table 1), with an unabsorbed $2-10 \mathrm{keV}$ flux of $1.10 \pm 0.04 \times 10^{-11} \mathrm{erg} \mathrm{cm}^{-2} \mathrm{~s}^{-1}$ (i.e., $\left.L_{\mathrm{x}}(2-10 \mathrm{keV}) \sim 10^{44} \mathrm{erg} \mathrm{s}^{-1}\right)$. It is consistent with the value found during the previous XMM-Newton observation in December 2000 (i.e., $1.09 \pm 0.01 \times 10^{-11} \mathrm{erg} \mathrm{cm}^{-2} \mathrm{~s}^{-1}$, Guainazzi 2003). There is still a clear positive deviation near $6.4 \mathrm{keV}$ (in the rest-frame), with this power-law model with $\Gamma \sim 1.77$ (which is the same index value as found in the $2-5 \mathrm{keV}$ energy range, see Fig. 1). The addition of a narrow (i.e., the line width $\sigma$ is fixed to $10 \mathrm{eV}$, i.e. intrinsically narrow) Gaussian emission line improves significantly the adjustment of the data $\left(\Delta \chi^{2}=30\right.$ for only two additional parameters, $F$-test probability of $99.99 \%$, see Table 1$)$. The rest-frame energy of the line at about $6.41 \mathrm{keV}$ corresponds to fluorescence from iron in a "low" state of ionization (i.e., $\leq$ Fe XVII) and its equivalent width $(E W)$ is consistent with the typical values measured in Seyfert 1 galaxies ( 50-150eV; e.g., Nandra \& Pounds 1994). We let free the line width, but we do not obtain strong constraint on this parameter with $\sigma<112 \mathrm{eV}$ (Table 1). Now we look for a possible weak broad line component, then we test a relativistic line model (Laor 1991). We fix the parameters of the line to those inferred from the RGS data for low- $Z$ ions O VIII, and $\mathrm{CVI} \mathrm{Ly}_{\alpha}$ (see Sect. 3.2). The line is badly fitted with only this relativistic line profile, then we add to the LAOR profile a narrow line component $(\sigma=10 \mathrm{eV})$. We obtain an upper limit for the $E W$ of the LAOR profile of $75 \mathrm{eV}$, and $E W$ of $61_{-27}^{+21} \mathrm{eV}$ for the narrow component. However the $\Delta \chi^{2}$ is less than unity for one additionnal parameter, comparing to the model with the narrow component line alone, see Table 1, therefore there is no statistical requirement for a broad component for the $\mathrm{Fe}_{\alpha}$ line at $6.4 \mathrm{keV}$ in the present observation. Similar results are obtained when using the relativistic line parameters at $6.4 \mathrm{keV}$ reported in Table 2 in Guainazzi (2003). We also test the possible presence of a line component at $5.7 \mathrm{keV}$ claimed by Guainazzi (2003) in the December 2000 XMM-Newton observation. Analysing the data of December 2000, we do not find any statistical requirement for a line at $5.7 \mathrm{keV}$ (rest-frame) with $E W<37 \mathrm{eV}$. As well this component is not required in the present January 2001 data with $E W<23 \mathrm{eV}$ and an $F$-test probability of only $40 \%$ (Table 1 ). The addition of a second narrow Gaussian emission line at higher energy $(E>6.4 \mathrm{keV})$ yields a further very slight improvement $\left(\Delta \chi^{2} \sim 5\right.$ for 2 additional parameters, $F$-test probability of $94.8 \%$, see Table 1). This second emission line is less well determined but it clearly lies to the high-energy side of the "cold" line with a best-fit energy $E=6.92 \mathrm{keV}$ (most probably due to FeXXVI, i.e. H-like iron). There is a negative deviation compared to the model near $7 \mathrm{keV}$ (in the galaxy frame), therefore we add an absorption edge and we obtain a better fit $\left(\Delta \chi^{2}=16\right.$ for 2 additional parameters, $F$-test probability of $99.97 \%$, Table 1$)$. Its energy is consistent with absorption due to cold to moderate ionized iron ions (i.e., <Fe XII). Figure 2 displays the contour plot for the optical depth of the edge versus its energy, for $\Delta \chi^{2}=2.3,4.61$, and $9.21(68 \%$, $90 \%$, and $99 \%$ confidence levels, respectively). The ionization state of the "cold" emission line and the absorption edge are compatible.

To summarize the present data are well represented in the $2-10 \mathrm{keV}$ energy range by a power-law and by a narrow $\mathrm{Fe} \mathrm{K}_{\alpha}$ line at about $6.4 \mathrm{keV}$, and a weak absorption edge 


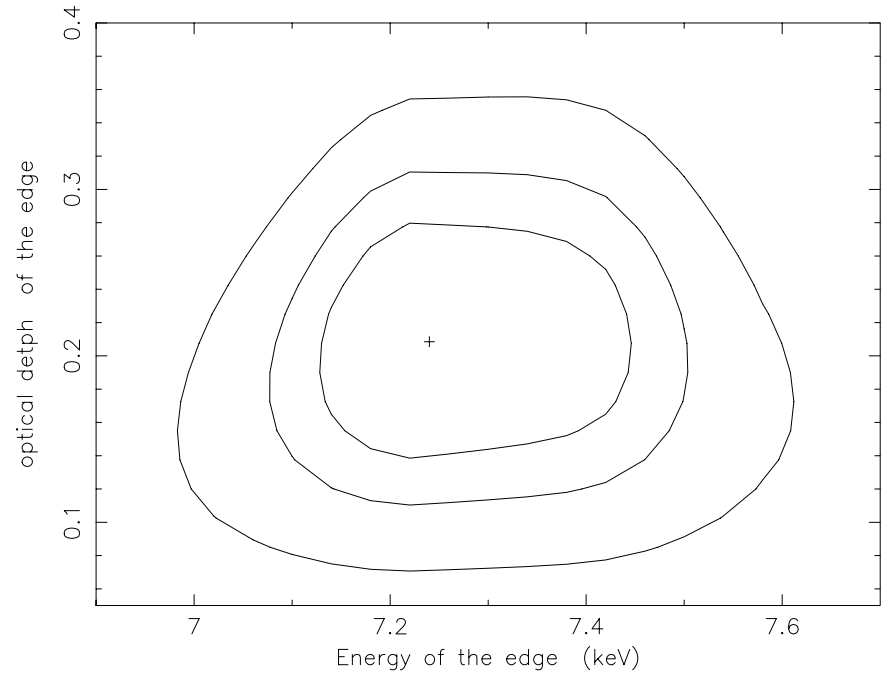

Fig. 2. The contour plot of the optical depth of the absorption edge versus its energy, for $\Delta \chi^{2}=2.3,4.61$, and $9.21(68 \%, 90 \%$, and $99 \%$ confidence levels, respectively).

near $7.2 \mathrm{keV}$. Both energies are compatible with iron nearly or moderatly ionized (i.e., <Fe XVII and $<$ Fe XII, respectively). Also there is a slight indication for a very ionized narrow $\mathrm{Fe}_{\alpha}$ emission line near $6.9 \mathrm{keV}$, which most probably corresponds to Fe XXVI, i.e. H-like iron. See Table 1 and Fig. 3.

The narrow component found here at $6.4 \mathrm{keV}$ is now emerging as a common feature of Seyfert 1 spectra seen by XMM-Newton and Chandra (see, e.g., Mrk 205: Reeves et al. 2001; NGC 5548: Yaqoob et al. 2001; NGC 3783: Kaspi et al. 2001). Detection of a significant narrow iron emission line at $6.4 \mathrm{keV}$ implies that a substantial quantity of cool reprocessing material is present. The line strength $(E W \sim 70 \mathrm{eV})$, implies that the cool matter if seen by reflection subtends a substantial solid angle, of at least 1 steradian, assuming it is Thomson thick and has a solar abundance of iron (e.g. George \& Fabian 1991). The most likely location of such material, according to the current AGN models, would appear to be the molecular torus (Antonucci 1993), with hard X-rays from the central engine being reflected off the inner surface of the torus and into the line-of-sight of the observer (Krolik et al. 1994; Ghisellini et al. 1994). Although reflection off the putative torus is attractive, we cannot rule out other options. Emission from BLR clouds could account for some of the narrow line flux as probably found in NGC 5548 (Yaqoob et al. 2001), although the predicted line strength is small $(E W<50 \mathrm{eV}$; Leahy \& Creighton 1993), but consistent within the error bars with the value found here. Another possibility is that the line originates by X-ray reflection from the outer regions of the accretion disc. Normally the angle subtended by the outer disk, to the X-ray source, would be small. However a warped, concave disk could increase the amount of reflection at large radii, and account for a measurable narrow line component at $6.4 \mathrm{keV}$ (Blackman 1999). The present $6.4 \mathrm{keV}$ emission line is much weaker than the one inferred from the ASCA observation in July 1997 (i.e. $E W=320_{-100}^{+70} \mathrm{eV}$ for an unresolved Gaussian line). As well, it also appears approximately two times weaker than the one measured during December 2000, by Guainazzi et al. (2003)

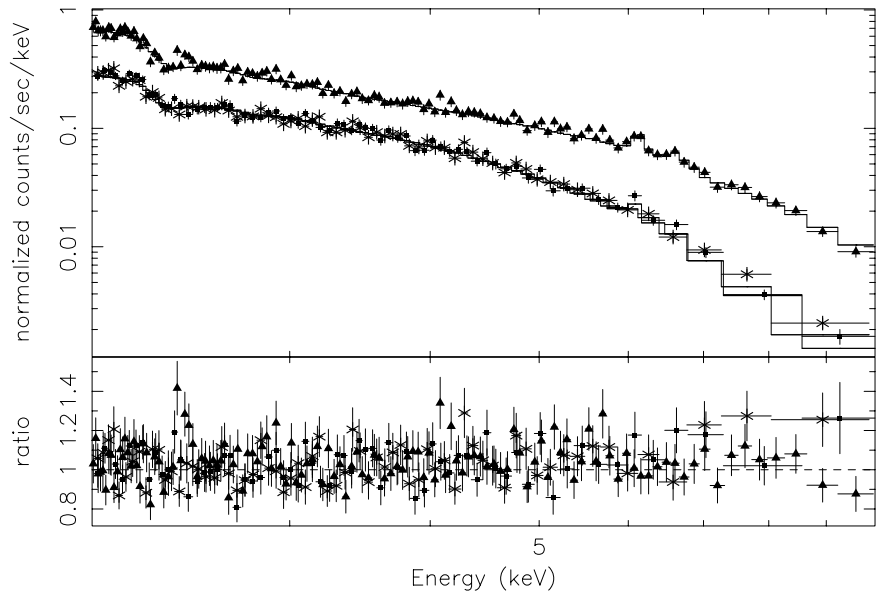

Fig. 3. The 2-10 keV EPIC spectrum of ESO 198-G24 (observer frame and binning of $10 \sigma$ ). An absorbed power-law, two Gaussian emission lines $(\sigma=10 \mathrm{eV})$ and one absorption edge have been fitted. Filled squares: MOS1, stars: MOS2, and triangles: PN.

with similar intrinsic $2-10 \mathrm{keV}$ fluxes. However the claim of a possible variability of this line between these two observations should be taken into caution, indeed analysing the December 2000 data (assuming a narrow line at $6.41 \mathrm{keV}$ and a line width of $10 \mathrm{eV}$ ), we obtain $E W=70 \pm 45 \mathrm{eV}$. This value is compatible with the one reported in Table 1 for the present data. No reliable comparison is possible with the BeppoSAX data, since its spectral resolution is not sufficient to determine whether the profile was narrow or broad, however the $E W$ are compatible within the error bars (i.e. $E W=100_{-60}^{+30} \mathrm{eV}$ ).

The emission line near $6.92 \mathrm{keV}$, if any, corresponding to Fe XXVI (H-like iron), could be produced by the disc matter in a high state of ionization, as observed in several radio-quiet quasars (Reeves \& Turner 2000). As we will show below, the EPIC data may be explained by an ionized accretion disk model from Ross \& Fabian (1993) and Ballantyne et al. (2001).

Another possibility is the ionized lines originate from the WA, which can produce substantial emission from $\mathrm{He}$ and $\mathrm{H}-$ like iron (Krolik \& Kallman 1987). As we will see below, the WA, if present, does not show significant absorption or emission features below $2.5 \mathrm{keV}$. This may mean that either there is only almost no warm absorber material on the line-of-sight, or the low- $\mathrm{Z}$ ions (e.g., $\mathrm{O}, \mathrm{Ne}, \mathrm{Mg}, \mathrm{Si}$ ) are completely ionized, which is consistent with the very high ionized state (H-like) found for the $\mathrm{Fe}_{\alpha}$ line at about $6.9 \mathrm{keV}$.

As reported by Guainazzi (2003), no absorption edges existed in the EPIC/PN spectrum during the observation of December 2000, with only an upper limit of 0.14 . However, the statistics of this observation is much lower than the one presented here, then enable any reliable comparison for a possible variability of the absorption edge. We notice that the absorption edge found in the EPIC data near $7.24 \mathrm{keV}$ (i.e. $<$ Fe XII) cannot be produced by the WA, if any. Indeed, the optical depth found for this absorption edge is about $0.1-0.2$, which means that the WA would be completly opaque below about $2 \mathrm{keV}$, while this is not what we demonstrate in Sect. 3.4. The WA for $\log \xi \leq 2$ corresponds to too weak column density to produce a significant absorption edge of cold iron ions as observed at $7.24 \mathrm{keV}$. 
Table 2. X-ray spectral fits over the $0.3-10 \mathrm{keV}$ broad band energy range. In all models we add two Gaussian emission lines and one absorption edge. The energies and the width of the lines are fixed to the values found in Table 1 for the last model. BB: black-body component. PL: powerlaw component. PEXRAV: exponentially cut off power-law spectrum reflected from neutral material (Magdziarz \& Zdziarski 1995), the cutoff energy has been fixed to $200 \mathrm{keV}$. COMPTT: comptonization model of soft photons in a hot plasma from Titarchuk (1994), here the soft photon temperature is set to be the same for the two hot plasmas. IONDISK: reflection spectrum from an ionized slab of an input power-law from Ross \& Fabian (1993) and Ballantyne et al. (2001). (a): the line fluxes are expressed in $10^{-6} \mathrm{erg} \mathrm{cm}^{-2} \mathrm{~s}^{1}$.

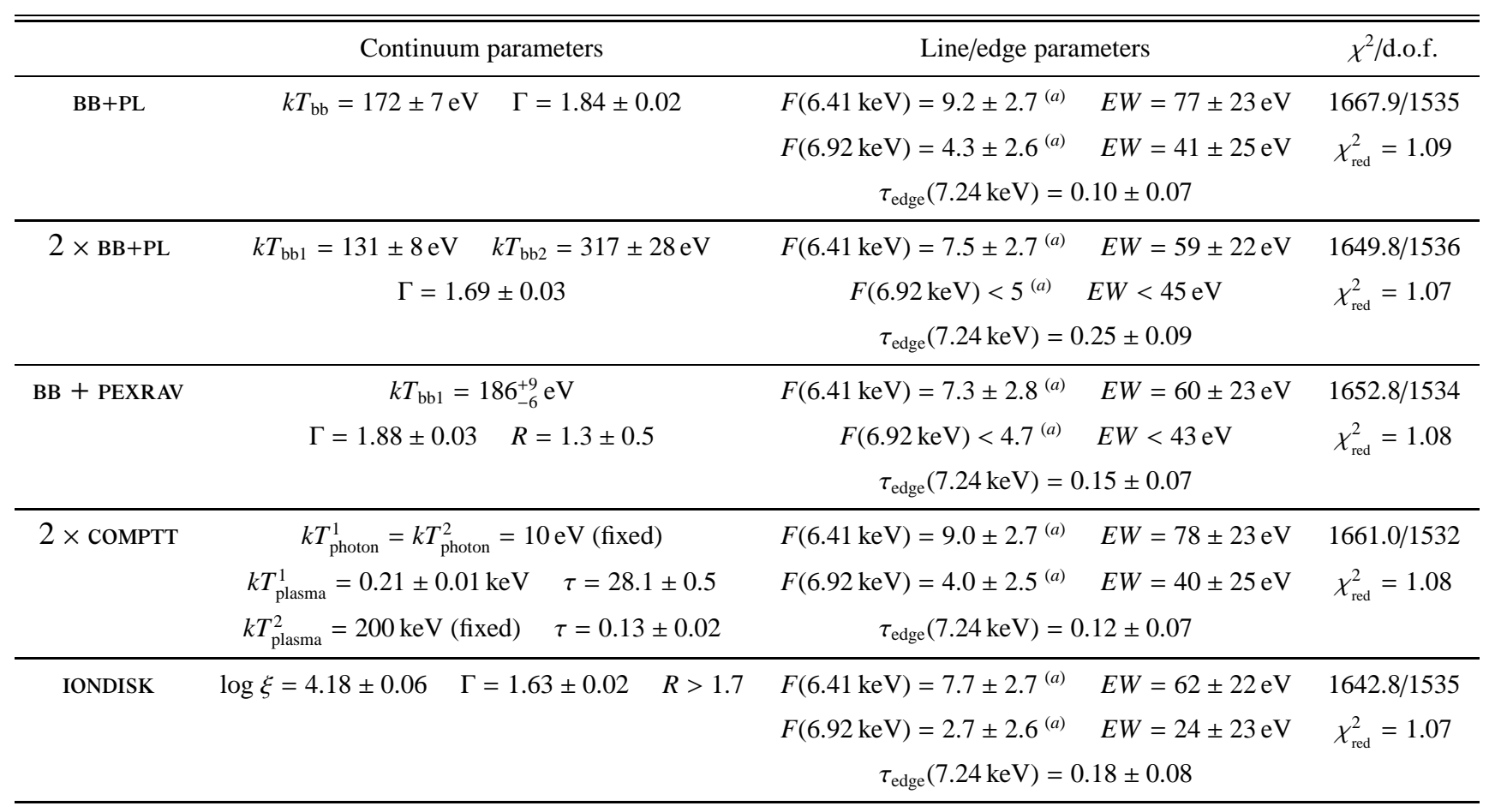

\subsubsection{The EPIC broad band spectrum}

Extrapolating the best-fitting $2-5 \mathrm{keV}$ power-law model back to $0.3 \mathrm{keV}$, both MOS and PN spectra clearly reveal the presence of a broad soft excess below about $1.5-2 \mathrm{keV}$ as shown in Fig. 1. This soft X-ray emission seems to be a common feature in Seyfert 1 galaxies observed up to now with XMMNewton (see Pounds \& Reeves 2002). The shape of this soft excess emission is similar to that found in other high-luminosity Seyfert 1 galaxies (see for example Fig. 1 in Pounds \& Reeves 2002).

Fitting the $0.3-2 \mathrm{keV}$ EPIC spectra of ESO 198-G24 by a single absorbed power-law model, we find a photon power-law index $\Gamma=1.94 \pm 0.02\left(\chi^{2} /\right.$ d.o.f. $\left.=592.6 / 558, \chi_{\text {red }}^{2}=1.06\right)$, much harder than those measured according to the two ROSAT observations, i.e. $\Gamma=2.28 \pm 0.07\left(\chi_{\text {red }}^{2}=2.52\right)$ and $\Gamma=$ $2.19 \pm 0.07\left(\chi_{\text {red }}^{2}=1.07\right)$ respectively for December 1991 and July 1992 (Turner et al. 1993). The unabsorbed $0.1-2 \mathrm{keV}$ $\mathrm{X}$-ray flux is $1.58 \pm 0.01 \times 10^{-11} \mathrm{erg} \mathrm{cm}^{-2} \mathrm{~s}^{-1}$. We now investigate different models which may explain the $0.3-10 \mathrm{keV}$ broad band EPIC spectra, i.e. both the soft excess feature and the hard spectrum. In each model, we take into account the iron complex found previously, we fix the energy of the two emission lines and of the absorption edge to the values found in Table 1 for the last model. The inferred parameters for all the following models are reported in Table 2. First, we fit the spectra with a black-body (soft X-rays) and with a power-law component (hard X-rays). We obtain a good fit for this combined model (see Table 2). Adding another black-body component improves the spectral fit (i.e. $\Delta \chi^{2}=18$ for 2 additional parameters). We also test other disk models such as DISKBB and DISKPN combined with a power-law, we obtain as well a good representation of the data, with $k T \sim 250 \mathrm{eV}$. The temperatures found here are rather large for a standard steady state $\alpha$ thin accretion disc. Assuming a black hole (BH) mass of about $3 \times 10^{8} M_{\odot}$ (Rokaki \& Boisson 1999), we expect a temperature of about only $10 \mathrm{eV}$ at $3 R_{\mathrm{s}}$ (e.g, Peterson 1997). Therefore a standard steady state $\alpha$ thin accretion disc is ruled out to explain the soft excess observed in ESO 198-G24.

The broad band X-ray spectrum of ESO 198-G24 may be explained by reflection from an accretion disc (e.g., PEXRAV, Magdziarz \& Zdziarski 1995). Since the energy range of our spectral fit is restricted, we fix the cut-off energy to $200 \mathrm{keV}$. This model gives a slightly poorer fit $\left(\chi^{2} /\right.$ d.o.f. $=1701.1 / 1536$, i.e. $\left.\chi_{\text {red }}^{2}=1.11\right)$ than the other ones reported in Table 2. Adding an emission component from the accretion disk (e.g., bb), we obtain a better fit with $\left(\Delta \chi^{2}=48\right.$ for 2 additional parameters, see Table 2). We obtain a reflection component close to 1.3 , i.e. is consistent with a reflection from a cold material with a $2.6 \pi$ steradian solid angle. However the black-body temperature is too high for a standard steady state $\alpha$ thin accretion disc as mentioned above. Therefore the $\mathrm{BB}+$ pexrav model is also ruled out.

Comptonization has often been suggested as a source of both the soft X-ray and hard X-ray spectrum of AGN, as for 

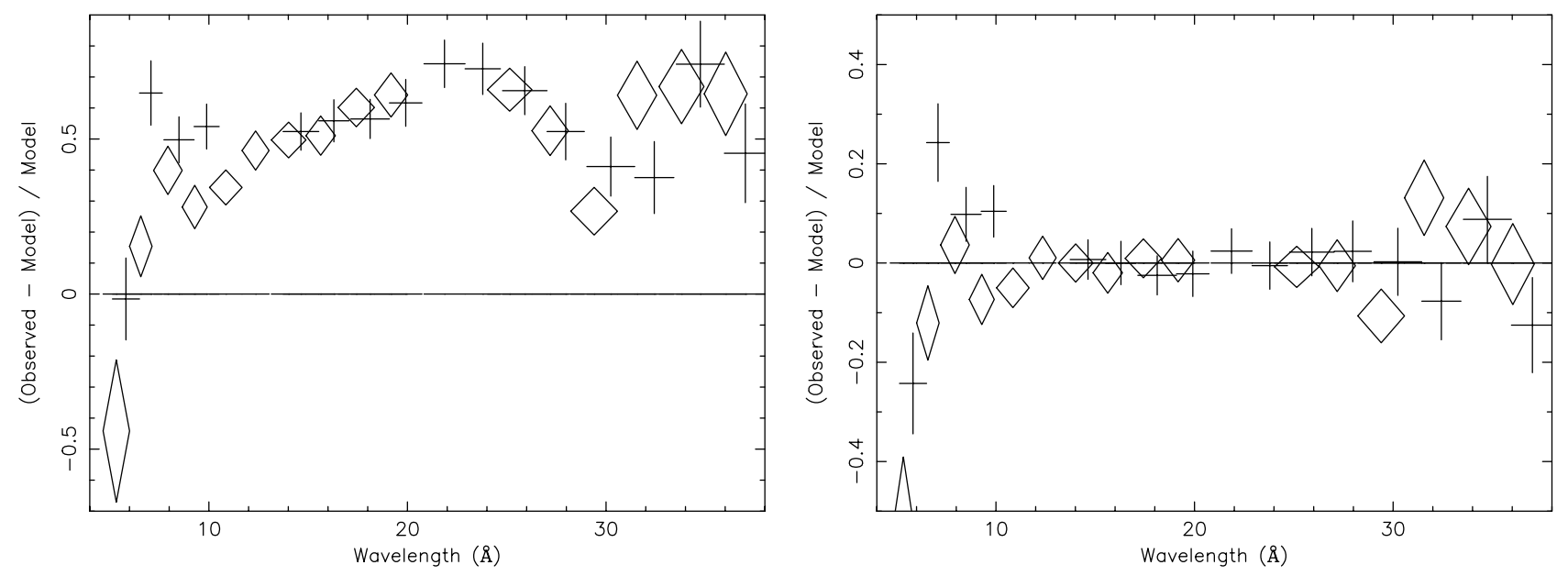

Fig. 4. The RGS fit residual spectra of ESO 198-G24 (observer frame) for the power-law (PL) model with the parameter fixed to the values found with EPIC. Left panel: PL only. Right panel: PL + "knak" model (see text for explanations). Crosses: RGS 1, diamonds: RGS 2.

example the accretion disc may be responsible for the soft emission and part of these soft photons are inversed-Compton scattered to the hard X-ray energy range, as they pass through the hot corona above the disc. We use a double Comptonization model (COMPTT: Titarchuk 1994) as in O'Brien et al. (2001) in which soft photons from the accretion disc are up-scattered by thermal electrons characterized by two temperatures, to yield the observed broad soft excess as well as the power-law shape at higher energies. We fix the temperature of the comptonizing photons at $10 \mathrm{eV}$ as expected for a $\mathrm{BH}$ mass of $3 \times 10^{8} M_{\odot}$ as mentioned above, and the plasma temperature to $200 \mathrm{keV}$ for the second COMPTT. The data are well fitted with this model (Table 2).

An ionized accretion disk model (Ross \& Fabian 1993; Ballantyne et al. 2001) provides also a good fit to the data with a very high ionization parameter $\left(\xi=L_{\mathrm{bol}} /\left(n_{\mathrm{H}} r^{2}\right)\right.$, with $L_{\mathrm{bol}}$ the bolometric luminosity in $\operatorname{erg~s}^{-1}, n_{\mathrm{H}}$ the hydrogen density of the ionized medium in $\mathrm{cm}^{-3}$, and $r^{2}$ the distance of the ionized medium from the photo-ionizing source in $\mathrm{cm}$ ). The value of $\xi$ found here is consistent with an emission of a H-like $\mathrm{Fe} \mathrm{K}_{\alpha}$ line (e.g., Fig. 6 from Colbert et al. 2002), and with the small residual found at $6.92 \mathrm{keV}$ in this model. The narrowness of this iron $\mathrm{H}$-like line indicates that the line is emitted far away from the $\mathrm{BH}$ or that the disk has a relatively small optically depth for Compton scattering. However, we note that the iondisk model used here does not take into account smearing of the emission lines and hence the derived ionisation parameter may be overestimated.

Two models can explain the broad band spectrum of ESO 198-G24: a double comptonization models or an ionized accretion disk.

\subsection{The RGS data analysis}

The RGS spectrum offers us for the first time a view of ESO 198-G24 at very high spectral resolution. While the spectrum has a rather poor $S / N$ ratio, we are able to test several models using the SPEX code (Kaastra et al. 2002a, version 2.0).
First, we start fitting the RGS 1 and RGS 2 spectra, with the EPIC best-fit parameters of the power-law model. The original spectrum was binned to two bins per CCD. The residuals show a clear soft excess (see Fig. 4 left panel). Then we modify the power-law model with the "knak" model which is a multiplicative model. It multiplies the spectral model by a factor $f(\lambda)$. The full wavelength range is divided into segments with bounding wavelengths $\lambda_{i}$. The multiplicative scaling factor $f_{i}$ for each segment boundary $\lambda_{i}$ is a free fitting parameter. For each wavelength the multiplication factor $f(\lambda)$ is determined by linear interpolation in the $\log \lambda_{i}-\log f_{i}$ plane. We have chosen the grid points $\lambda_{i}$ at $6,12,18,24,30$ and $36 \AA$. This empirical model gives a much better fit with $\chi^{2}=51.8$ for 26 d.o.f. (see Fig. 4 right panel). Half of the $\chi^{2}$ value is due to the shortest wavelength bin of each RGS, and this is mainly due to remaining calibration uncertainties below $\sim 7 \AA$. We obtain that the source as for example a soft excess amplitude of $66 \%$ at $36 \AA$, and $44 \%$ at $12 \AA$.

Evident absorption/emission features are not visible. Therefore, if there is a WA, it either has a relatively low column density, or a very high ionization parameter $\left(\xi=L /\left(n_{\mathrm{H}} r^{2}\right)\right)$. The WA is an optically thin ionized medium, detected in most Seyfert 1 galaxies with low to moderate X-ray luminosities $\left(L_{\mathrm{X}}<10^{44} \mathrm{erg} \mathrm{s}^{-1}\right)$. Up to now, RGS spectra confirm the absence of strong absorption in the high luminosity sources (e.g., Mrk 509: Pounds et al. 2001; PKS 0558-504; O'Brien et al. 2001). Low density warm absorbers are most easily detected from their narrow absorption lines (see for example Kaastra 2001). To test the presence of warm absorbing gas, we therefore use the above empirical continuum model (PL + knak) and apply the "xabs" WA model (see Kaastra et al. 2002b). The velocity broadening of the absorber was fixed to $\sigma_{v}=100 \mathrm{~km} \mathrm{~s}^{-1}$; since we expect low column density, the equivalent line width does not depend upon $\sigma_{v}$ but merely upon the column density. We use the spectrum with a binning factor of 3 . We search a grid of ionisation parameters $(\log \xi$ from -2 to +3.5$)$ with a set of outflow velocities: $v=0,-250,-500,-1000$ and $-2000 \mathrm{~km} \mathrm{~s}^{-1}$, but found no clear detection of a WA. The $1 \sigma$ upper limits to the column densities are shown in Fig. 5. If any 


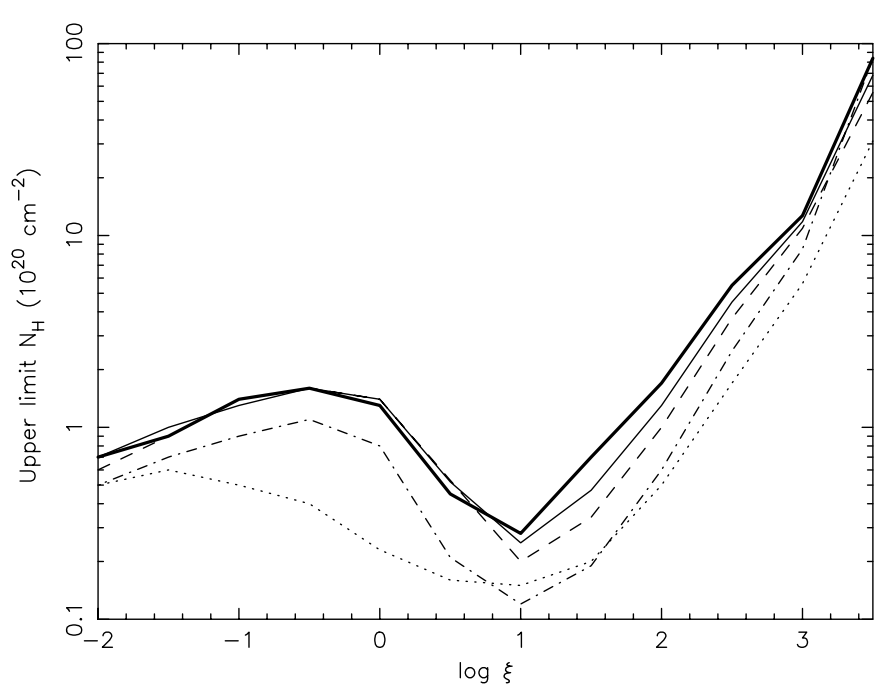

Fig. 5. Upper limit at $1 \sigma$ of the column density $\mathcal{N}_{\mathrm{H}}$ (expressed in $10^{20} \mathrm{~cm}^{-2}$ ), versus the ionization parameter $\xi$ (in logarithm scale). The curves correspond to different values of outflow velocities. Thick solid line: $v=0 \mathrm{~km} \mathrm{~s}^{-1}$, thin solid line: $v=-250 \mathrm{~km} \mathrm{~s}^{-1}$, dashed line: $v=-500 \mathrm{~km} \mathrm{~s}^{-1}$, dash-dot line: $v=-1000 \mathrm{~km} \mathrm{~s}^{-1}$, and dots: $v=-2000 \mathrm{~km} \mathrm{~s}^{-1}$.

WA is present, its column density is low for $\log \xi \leq 2$. Even in case of a very highly ionised medium (i.e., here higher column density upper limit), the low- $Z$ ions which absorbed in the soft X-ray range are completely ionised, then in both cases (moderately or very highly ionised medium) we do not expect strong absorption edges. We also can put limits on narrow line emission: the best-fit $\mathrm{O}$ VII $f$ (forbidden) line has a strength of $<3.2 \times 10^{50} \mathrm{ph} \mathrm{s}^{-1}$, the best-fit Ne IX $f$ line has a strength of $0.4 \pm 0.8 \times 10^{50} \mathrm{ph} \mathrm{s}^{-1}$ Thus, no significant narrow line emission is found.

As a last step, we investigate the broad continuum emission features. We find a best-fit model using the combination of a power-law, a modified black body continuum (MBB), and weak

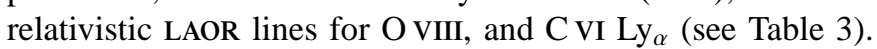
The power-law parameters are kept fixed to the EPIC values. Since there are no narrow features in the spectrum, we bin the spectrum by a factor of 100 for the spectral fitting. Figure 6 displays the average RGS spectrum, with superposed the contribution of the power-law, the power-law plus modified black body continuum (MBB), and the best fit model including all components. The Laor line profiles are intrinsically very broad and asymmetric, and moreover, they are folded with the instrumental effective area, which also contains significant broad band structure. The dotted line in Fig. 6 displays the sum of the C VI and $\mathrm{O}$ VIII contributions, and due to the cosmological redshift, the line spectrum is shifted by about $5 \%$ or $1-2 \AA$ for the relevant part. The bright bin around $24 \AA$ is the red wing of the O VIII line (not the peak of a N VII line), the part between 28 and $32 \AA$ is a combination of the reddest part of O VIII line with the blue wing of the C VI line, and the part above $32 \AA$ is dominated by the red wing of the $\mathrm{C}$ VI line.

To summarize, we find that the RGS spectrum is consistent with an interpretation in terms of weak relativistic lines. The formal significance of the O VIII and C VI lines is $3.6 \sigma$
Table 3. Best-fit parameters for the average RGS spectrum with a model combining a power-law, a modified black body continuum (MBB), and relativistic LAOR lines for O VIII, N VII and C VI Ly ${ }_{\alpha}$. The power-law parameters are kept fixed to the EPIC values.

\begin{tabular}{cc}
\hline \hline$\chi^{2} /$ d.o.f. & $61.85 / 42$ \\
\hline O VIII & norm $=62 \pm 17 \times 10^{50} \mathrm{ph} \mathrm{s}^{-1}$ \\
$(\lambda=18.97 \AA)$ & $E W=2.2 \pm 0.6 \AA$ \\
N VII & norm $=0 \pm 15 \times 10^{50} \mathrm{ph} \mathrm{s}^{-1}$ \\
$(\lambda=24.78 \AA)$ & $E W=0.0 \pm 0.6 \AA$ \\
C VI & norm $=118 \pm 41 \times 10^{50} \mathrm{ph} \mathrm{s}^{-1}$ \\
$(\lambda=33.74 \AA)$ & $E W=5.5 \pm 1.9 \AA$ \\
inclination & $60^{\circ}{ }_{-11}^{+5}$ \\
$q$ & $q>5.4$ \\
$r_{1}$ (in GM $\left./ \mathrm{c}^{2}\right)$ & $1.64_{-0.14}^{+0.45}$ \\
$r_{2}$ (in GM $\left./ \mathrm{c}^{2}\right)$ & $400($ fixed $)$ \\
\hline MBB & $0.53 \pm 0.06 \mathrm{keV}$ \\
\hline & norm $=0.58 \pm 0.17 \times 10^{32} \mathrm{~m}^{0.5}$ \\
\hline
\end{tabular}

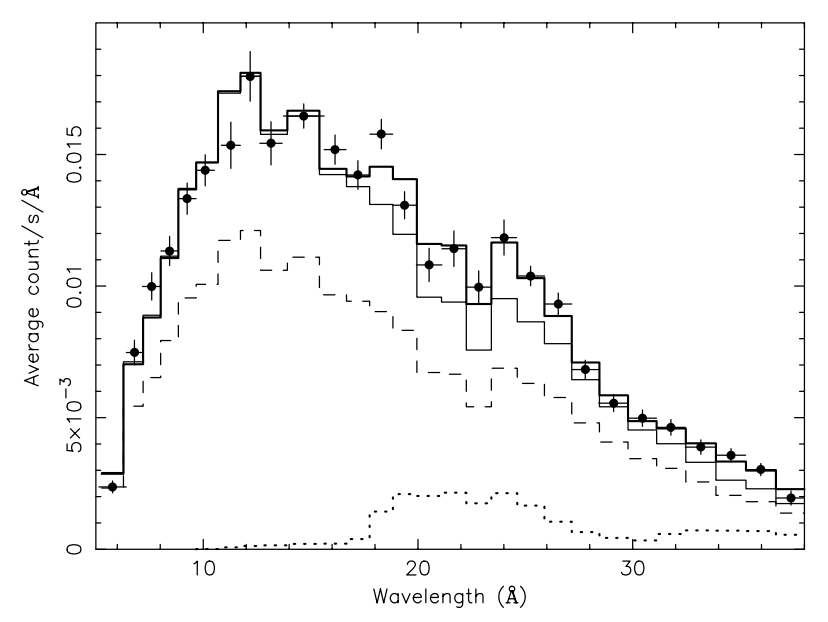

Fig. 6. Average RGS spectrum (points), with superposed different components: the PL (dashed line), the relativistic LAOR lines (dotted line), PL+MBB (thin solid line), and the best-fit model (thick solid line): PL + MBB + relativistic LAOR lines for O VIII, N VII and $\mathrm{C}$ VI $\mathrm{Ly}_{\alpha}$.

and $2.9 \sigma$, respectively. We do not exclude alternative spectral models, but for example an interpretation in terms of a dusty warm absorber giving deep edges at the wavelengths of the blue edges of our broad lines is difficult given the lack of deep narrow absorption lines in the spectrum (see also our tight limits on the column density of any warm absorber in Fig. 4).

\section{Summary and discussion}

The present data analysis of the observation of ESO 198-G24 with XMM-Newton (January 24, 2001), has revealed that this 
Seyfert 1 has an unabsorbed $2-10 \mathrm{keV}$ luminosity of about $1.1 \times$ $10^{-11} \mathrm{erg} \mathrm{s}^{-1}$.

We found both with EPIC and RGS that this source possesses a significantly steeper spectrum below $\sim 1.5-2 \mathrm{keV}$ than observed at higher X-ray energies, the so-called soft X-ray excess. This soft X-ray emission seems to be a common feature in Seyfert 1 galaxies observed up to now with XMM-Newton (see Pounds \& Reeves 2002). The shape of this soft excess emission is similar to those found in other high-luminosity Seyfert 1 galaxies. (see for example Fig. 1 in Pounds \& Reeves 2002). The RGS spectra reveal no significant narrow absorption lines suggesting that if there is a warm absorber, it either has a relatively low column density, or a very high ionization parameter. The RGS data are well described by the combination a power-law, a modified black body continuum, and weak relativistic soft X-ray lines from $\mathrm{H}$-like ions O VIII, and $\mathrm{CVI} \mathrm{Ly}_{\alpha}$, as may be observed with the RGS in at least two others Seyfert 1 galaxies: Mrk 766 and MCG-6-30-15 (Branduardi-Raymont et al. 2001; Mason et al. 2003). However in our case the equivalent width of the relativistic lines is a factor of 5-10 smaller than in the two sources mentioned above. The presence of relativistic soft X-ray lines in AGN is a hotly debated issue (e.g., Lee et al. 2001; Branduardi-Raymont et al. 2001; Mason et al. 2003). Here is not the place to discuss this issue in detail, but we note that the strength of the proposed relativistic lines in our data are consistent with those expected from ionized disk models, while the absence of strong absorption lines makes the alternative dusty warm absorber less likely. However other interpretations are not definitely excluded.

The EPIC data displayed a narrow emission line at about $6.4 \mathrm{keV}$, corresponding to low or moderate ionized iron (i.e., $\leq$ Fe XVII). We find no significant broad (relativistic line) component, if any in our observation, with only an upper limit of $75 \mathrm{eV}$. Then the possible contribution from the inner part of an accretion disk where the gravitational effects of the $\mathrm{BH}$ are strong, is weak in the present XMM-Newton observation. We find for the narrow line a moderate equivalent width of about $60-70 \mathrm{eV}$. The narrow line at $6.4 \mathrm{keV}$ seems to be an increasingly common feature in the higher quality spectral data emerging from Chandra and XMM-Newton (e.g., Mrk 509: Pounds et al. 2001, NGC 5548: Yaqoob et al. 2001, Mrk 205: Reeves et al. 2001). The emission medium responsible for the line component at $6.4 \mathrm{keV}$ can be find with search of possible response to continuum variation with high statistical data: inner part of the nucleus (accretion disc), BLR (as in NGC 5548), NLR, and molecular torus (as in Mrk 509 and Mrk 205), etc. The very weak ionized line associated to Fe XXVI, if any, could be interpreted in terms of fluorescence from $\mathrm{H}$-like iron in the highly ionized inner disk material, or could also be the signature of a very ionized WA. While we show that the absorption edge found near $7.24 \mathrm{keV}$ cannot be produced by the WA.

Acknowledgements. This work is based on observations obtained with XMM-Newton, an ESA science mission with instruments and contributions directly funded by ESA Member States and the USA (NASA). The authors would like to thank the anonymous referee for careful reading of the manuscript. D.P. acknowledges grant support from MPE fellowship (Germany). The Space Research Organisation of the Netherlands (SRON) is supported financially by NWO, the Netherlands Organisation for Scientific Research. Support from a PPARC studentship is acknowledged by KLP.

\section{References}

Anders, E., \& Grevesse, N. 1989, Geochim. Cosmochim. Acta, 53, 197

Antonucci, R. 1993, ARA\&A, 31, 473

Ballantyne, D. R. Iwasawa, K., \& Fabian, A. C. 2001, MNRAS, 323, 506

Blackman, E. G. 1999, MNRAS, 306, L25

Branduardi-Raymont, G., Sako, M., Kahn, S. M., et al. 2001, A\&A, 365, L140

Colbert, E. J. M., Weaver, K. A., Krolik, J. H., Mulchaey, J. S., \& Mushotzky, R. F. 2002, ApJ, 581, 182

den Herder, J. W., Brinkman, A. C., Kahn, S. M., et al. 2001, A\&A, 365, L7

George, I. M., \& Fabian, A. C. 1991, MNRAS, 249, 352

Ghisellini, G., Haardt, F., \& Matt, G. 1994, MNRAS, 267, 743

Guainazzi, M. 2003, A\&A, 401, 903

Kaastra, J. S. 2001, X-ray Observations of AGN, in Spectroscopic Challenges of Photoionized Plasmas, ASP Conf. Ser., 247, 39

Kaastra, J. S., Mewe, R., \& Raassen, A. J. J. 2002a, in New Visions of the X-ray Universe in the XMM-Newton and Chandra Era, ed. F. A. Jansen, ESA, in press

Kaastra, J. S., Steenbrugge, K. C., Raassen, A. J. J., et al. 2002b, A\&A, 386, 427

Kaspi, S., Brandt, W. N., Netzer, H., et al. 2001, ApJ, 554, 216

Krolik, J. H., \& Kallman, T. R. 1987, ApJ, 320, L5

Krolik, J. H., Madau, P., \& Zycki, P. T. 1994, ApJ, 420, L57

Laor, A. 1991, ApJ, 376, 90

Leahy, D. A., \& Creighton, J. 1993, MNRAS, 263, 314

Lee, J. C., Ogle, P. M., Canizares, C. R., et al. 2001, ApJ, 554, L13

Magdziarz, P., \& Zdziarski, A. A. 1995, MNRAS, 273, 837

Malizia, A., Bassani, L., Zhang, S. N., et al. 1999, ApJ, 519, 637

Mason, K. O., Branduardi-Raymont, G., Ogle, P. M., et al. 2003, ApJ, 582,95

Nandra, K., \& Pounds, K. A. 1994, MNRAS, 268, 405

O’Brien, P. T., Reeves, J. N., Turner, M. J. L., et al. 2001, A\&A, 365, L122

Peterson, B. M. 1997, An introduction to active galactic nuclei (New York: Cambridge University Press)

Piccinotti, G., Mushotzky, R. F., Boldt, E. A., et al. 1982, ApJ, 253, 485

Pounds, K. A., Nandra, K., Stewart, G. C., George, I. M., \& Fabian, A. C. 1990, Nature, 344, 132

Pounds, K., Reeves, J. N., O'Brien, P. T., et al. 2001, ApJ, 559, 181

Pounds, K., \& Reeves, J. 2002, to appear in New Visions of the X-ray Universe in the XMM-Newton and Chandra Era, 26-30 November 2001, ESTEC, The Netherlands [astro-ph/0201436]

Reeves, J. N., \& Turner, M. J. L. 2000, MNRAS, 316, 234

Reeves, J. N., Turner, M. J. L., Pounds, K. A., et al. 2001, A\&A, 365, L134

Rokaki, E., \& Boisson, C. 1999, MNRAS, 307, 41

Ross, R. R., \& Fabian, A. C. 1993, MNRAS, 261, 74

Strüder, L., Briel, U., Dennerl, K., et al. 2001, A\&A, 365, L18

Tanaka, Y., Nandra, K., Fabian, A. C., et al. 1995, Nature, 375, 659

Titarchuk, L. 1994, ApJ, 434, 570

Turner, T. J., George, I. M., \& Mushotzky, R. F. 1993, ApJ, 412, 72

Turner, M. J. L., Abbey, A., Arnaud, M., et al. 2001, A\&A, 365, L27

Wilms, J., Allen, A., \& McCray, R. 2000, ApJ, 542, 914

Wilms, J., Reynolds, C. S., Begelman, M. C., et al. 2001, MNRAS, 328, L27

Yaqoob, T., George, I. M., Nandra, K., et al. 2001, ApJ, 546, 759 